Introduction Different treatment strategies employed for endovascular thrombectomy (EVT) may impact successful reperfusion and functional outcome. The ASSIST Registry is a postmarket observational study for continued evaluation of new products per their intended use. The aim of the ASSIST registry is to collect real-world data to develop clinical evidence regarding the use of various techniques of EVT in large vessel occlusions (LVOs). Analysis include evaluating which strategies are associated with first pass reperfusion and better clinical outcomes.

Methods Prospective, global, consecutive enrollment registry (up to 1500 subjects) of acute ischemic stroke patients (AIS) with LVO treatment in anterior circulation treated with multiple interventional techniques [Stentriever + Balloon guide catheter (BGC); Stentriever + Aspiration \pm BGC; Aspiration \pm BGC] using Stryker Neurovascular devices for the first pass. Patients will be distributed in each arm with accommodations made for reducing heterogeneity by geographical and operator location. The data from ASSIST will be analyzed using a generalized linear mixed model which will employ a binary distribution and logit link function to predict mRS. The model will accommodate any categorical and continuous variables that are shown to be confounders by separate univariate analyses, will include a random effect for site, and a four-level variable denoting the technique type.

Results A total of 1198 patients have been enrolled to date across 48 global centers. Severity of disability (90-day mRS 02 ) and procedural outcome (eTICI $2 c$ or greater on first pass as adjudicated by core lab) will be evaluated for each technique. Secondary clinical outcomes include NIHSS drop of $\geq 10$ points from baseline or NIHSS score of 0 or 1 . Safety outcomes include mortality, neurological deterioration, symptomatic intracerebral hemorrhage (ICH) and embolization to a new territory. Baseline, follow-up and angiographic outcomes will be core lab adjudicated.

Conclusion There is limited evidence demonstrating clinical benefit or impact on outcomes based on the treatment strategy being employed to treat LVO with EVT. The ASSIST Registry will collect global real-world benchmark data on a large AIS population using the most common techniques and most recently available devices. Study results will provide valuable information on the relative effectiveness of different EVT treatment techniques and aid in the identification of optimal treatment approaches.

Disclosures R. Gupta: 1; C; Stryker Neurovascular PI ASSIST Registry, Zoll PI RECCLAIM II (No compensation), Cerenovous Steering Committee MEMBRANE study, Medtronic Steering Committee ELEVATE Study, Penumbra CEC MIND Trial, Vesalio PI CLEAR Study, Rapid Medical PI Tiger Study. A. Rai: 2; C; Stryker Neurovascular. D. Liebeskind: 2; C; Cerenovous, Stryker, Genentech, Medtronic, Rapid Medical. A. Krajina: 2; C; Stryker Neurovascular. M. Psychogios: None. T. Krings: None. W. Yoon: None. O. Zaidat: 1; C; Penumbra, Stryker Neurovascular. 2; C; Stryker Neurovascular, Penumbra, Rapid Medical, Cerenovous, Medtronic. A. Puri: 2; C; Stryker Neurovascular, Medtronic. A. Sarraj: 1; C; Stryker Neurovascular. 2; C; Stryker Neurovascular. M. Möhlenbruch: 2; C; Stryker Neurovascular, Phenox, Codman, Medtronic, Microvention.

\section{E-003 SAFETY AND EFFICACY OF BALLOON GUIDE CATHETERS IN THROMBECTOMY FOR ANTERIOR CIRCULATION STROKE: A SYSTEMATIC REVIEW AND META-ANALYSIS}

${ }^{1} \mathrm{~N}$ Shlobin*, 'B Jahromi, ${ }^{2} \mathrm{~S}$ Ansari, , $\mathrm{M}$ Potts. ${ }^{1}$ Neurological Surgery, Feinberg School of Medicine, Chicago, IL; ${ }^{2}$ Radiology, Feinberg School of Medicine, Chicago, IL

\subsection{6/neurintsurg-2021-SNIS.99}

Objective The benefit of balloon guide catheters (BGCs) in mechanical thrombectomy (MT) remains unclear. We examined the effect of BGC on procedural, radiographic, and clinical outcomes.

Methods A systematic review was conducted using PubMed, Embase, and Scopus to identify studies comparing MT for acute ischemic stroke with and without the use of BGCs. Patient demographics, procedural metrics, and outcomes were abstracted. Three-month functional outcomes were based on the modified Rankin Score (mRS).

Results Of the 2,181 resultant articles identified by the systematic review, 9 met inclusion criteria, comprising 2292 BGC and 1868 non-BGC patients. At baseline, the BGC group had a higher incidence of atrial fibrillation $(43.3 \%$ versus $36.2 \%, \mathrm{p}<0.0001$ ) and a lower National Institutes of Health Stroke Scale (NIHSS) score (15.8 versus 16.5, $\mathrm{p}=0.046)$. BGC use was associated with shorter groin-torecanalization times (55.6 versus 73.7 minutes, $\mathrm{p}=0.003$ ), improved TICI $\geq 2 \mathrm{~b}$ reperfusion $(83.8 \%$ versus $75.6 \%$, $\mathrm{p}<0.0001)$, lower incidence of symptomatic intracerebral hemorrhage (sICH; 5\% versus 7.7\%, p=0.046), and lower mortality $(16.4 \%$ versus $22.3 \%, \mathrm{p}<0.0001)$. BGC use, however, was also associated with a higher number of passes (2 versus 1.3, p<0.0001). There was no difference in functional outcomes at 90 days.

Conclusion BGC use during MT for acute ischemic stroke is associated faster to groin-to-recanalization times, improved TICI $\geq 2 b$ reperfusion, lower incidence of sICH, and lower mortality. These data demonstrate the promise of BGC use for MT and warrant further study.

Disclosures N. Shlobin: None. B. Jahromi: None. S. Ansari: None. M. Potts: None.

\section{E-004 FOCAL INTRACRANIAL VASCULOPATHY AS A MANIFESTATION OF COVID-19-ASSOCIATED ACUTE ISCHEMIC STROKE}

A Mahapatra*, A Witek, G Toth. Cerebrovascular Center, Cleveland Clinic Foundation, Cleveland, $\mathrm{OH}$

\subsection{6/neurintsurg-2021-SNIS.100}

Introduction COVID-19 infection has been associated with an increased risk of thrombotic events, including cerebrovascular accidents, presumed to be secondary to a systemic hypercoagulable state. These events have been reported even in young patients, without other significant vascular risk factors. We present a different, atypical case of a large-vessel occlusion (LVO) acute ischemic stroke secondary to a focal vasculopathy in a young patient with COVID-19 infection, requiring mechanical thrombectomy and emergent intracranial stenting, and we also review available literature. 
Methods Case analysis and literature review.

Results A patient in their early 20's with mild obesity presented to the emergency department (ED) one hour after acute onset of left hemiplegia (NIHSS 11). Emergent imaging revealed multifocal right middle cerebral artery territory acute ischemic infarcts with small petechial hemorrhage. CT angiography showed a right M1 segment occlusion. He was not a candidate for intravenous thrombolysis. Patient underwent mechanical thrombectomy with contact aspiration. The occluded right M1 segment was successfully recanalized, but follow-up angiography revealed re-stenosis. Balloon angioplasty was performed, but repeat angiography again demonstrated critical re-stenosis.. A balloon-mounted stent was placed in the R M1 segment, with successful recanalization and no further restenosis. The patient was acutely loaded with intravenous tirofiban, followed by oral aspirin and clopidogrel. Notably, the patient tested positive for COVID-19, but remained otherwise asymptomatic. Laboratory investigation, including hypercoagulabe and autoimmune workup for typical and atypical stroke etiologies, did not reveal any significant abnormalities, but the patient did have mildly elevated d-Dimer, and a minimally elevated homocysteine levels. Lower extremity ultrasound was negative for deep venous thrombosis, and echocardiogram was negative for significant abnormalities or intracardiac shunts. No cardiac arrhythmia was found. Patient was discharged home on hospital day five with NIHSS 1 (mild left facial droop) on aspirin and clopidogrel. At a two-month follow-up, patient remained without any objective residual deficits. Transcranial Doppler ultrasound at follow-up revealed full patency of the intracranial stent. Our literature search revealed a large body of evidence for acute stroke, LVO and secondary hypercoagulable state in COVID patients. However, focal vasculopathy, occasionally described with other viral infections such as $\mathrm{VZV}$, has not been reported in association with COVID infection.

Conclusion To our knowledge, our case is the first to illustrate the potential for COVID-19 infection to present as a focal intracranial vasculopathy in an otherwise healthy youngpatient, resulting in acute ischemic stroke without an underlying hypercoagulable state. Rescue intracranial stenting was necessary to maintain vessel patency and restore intracranial flow. Disclosures A. Mahapatra: None. A. Witek: None. G. Toth: None.

\section{E-005 PRELIMINARY RESULTS OF THE SUMMIT NZ ISCHEMIC STROKE TRIAL: MONOPOINT ${ }^{\circledR}$ EMBOLECTOMY FEATURING A NOVEL 088 ASPIRATION CATHETER AND SPECIALIZED DELIVERY CATHETER}

\footnotetext{
${ }^{1} S$ Brew*, ${ }^{1} B$ McGuinness, ${ }^{1} \mathrm{~J}$ Caldwell, ${ }^{1} S$ Lee, ${ }^{2} \mathrm{P}$ Barber, ${ }^{3} \mathrm{~A}$ Holden, ${ }^{4} \mathrm{~T}$ Wu, ${ }^{5} \mathrm{M}$ Krauss, ${ }^{5} \mathrm{~A}$ Laing, ${ }^{5} \mathrm{~W}$ Collecutt, ${ }^{6} \mathrm{D}$ Liebeskind, ${ }^{7} \mathrm{~S}$ Hetts. 'Interventional Neuroradiology, Auckland City Hospital, Auckland, New Zealand; ${ }^{2}$ Neurology, Auckland City Hospital, Auckland, New Zealand; ${ }^{3}$ Interventional Radiology, Auckland City Hospital, Auckland, New Zealand; ${ }^{4}$ Neurology, Christchurch Hospital, Christchurch, New Zealand; ${ }^{5}$ Interventional Radiology, Christchurch Hospital, Christchurch, New Zealand; ${ }^{6}$ Neurology, UCLA, Los Angeles, CA; ${ }^{7}$ Radiology, UCSF, San Francisco, CA
}

Introduction First-line aspiration is becoming a preferred technique for acute ischaemic stroke treatment. Recent technical advances that facilitate successful first-pass aspiration include larger bore embolectomy catheters guided by more manoeuvrable delivery catheters.

Materials A novel tapered tip delivery catheter was specifically designed to deliver an 0.088 " aspiration catheter through a specialized $8 \mathrm{~F} 90 \mathrm{~cm}$ introducer sheath (Route 92 Medical, Inc. San Mateo, CA) to M1 and ICA/ICA-T occlusions. The unique operating system, MonoPoint ${ }^{\circledR}$, is designed to maximize aspiration physics and improve first pass efficiency by engulfing the embolus. Utilizing a single RHV and a novel catheter-on-a-rail simplifies operator handling and control. Tenzing ${ }^{\circledR}$, the specialized delivery catheter, replaces the traditional microcatheter and is designed to reduce the need for a microwire to facilitate catheter delivery to the face of the embolus.

Methods SUMMIT NZ (ACTRN12619000890134p) was designed as a prospective, single-arm, open label clinical trial at two sites in New Zealand. Eligible participants were patients presenting with acute ischemic stroke from either anterior or posterior circulation large-vessel occlusion within 24 hours of onset, a National Institutes of Health Stroke Scale Score of greater than or equal to 6 and a pre-stroke modified Rankin Score of less than or equal to 2 . The primary effectiveness endpoint was arterial revascularization as measured by a modified Thrombosis in Cerebrovascular Infarction (mTICI) score of $2 \mathrm{~b}$ or greater at the end of angiography after all endovascular treatments as adjudicated by an independent core laboratory. The primary safety endpoints were device-related peri-procedural complications such as dissection or perforation, symptomatic Intracranial Cerebral Hemorrhage (SICH) at 24 hours and embolization to a previously uninvolved territory.

Results From September 27, 2019 to January 11, 2021, 34 subjects (mean age 68.5, NIHSSS 15.9, time last known well 5.9 hours) were enrolled with a diagnosis of acute ischemic stroke. Acute occlusion was located in the middle cerebral artery $(74 \%, 25 / 34)$ and internal carotid artery $(26 \%, 9 / 34)$. Successful revascularization was achieved in 97\% (33/34) of subjects. No serious adverse device effects have been reported. Conclusions Preliminary findings suggest that first-line aspiration embolectomy using a novel MonoPoint ${ }^{\circledR}$ system including an 0.088 " inner diameter aspiration catheter achieves a high rate of arterial revascularization with an acceptable safety profile. A full report on enrollment and results are to be presented.

Disclosures S. Brew: 1; C; Route 92 Medical. 2; C; Route 92 Medical, Microvention. B. McGuinness: 1; C; Route 92 Medical. 2; C; Route 92 Medical. J. Caldwell: 1; C; Route 92 Medical. 2; C; Route 92 Medical. S. Lee: 1; C; Route 92 Medical. P. Barber: None. A. Holden: 2; C; Medtronic, Gore, Boston Scientific. T. Wu: None. M. Krauss: None. A. Laing: None. W. Collecutt: None. D. Liebeskind: 2; C; Cerenovus, Genentech, Medtronic, Rapid Medical, Stryker. S. Hetts: 2; C; Stryker, Microvention, Terumo, ThrombX. 